\title{
Effect of pan-caspase inhibitor in ex vivo cold ischemia-rewarming injury model
}

\author{
Won-Hee Cho, Jung-Woo Seo, Su Woong Jung, Sang-Ho Lee
}

Division of Nephrology, Department of Internal Medicine, Kyung Hee University Hospital at Gangdong, Seoul, Korea

Background: Although the mouse model of vascularized kidney transplantation is a powerful tool for investigating the mechanisms of cold ischemia-reperfusion injury (IRI), the surgery is technically difficult. Here, we evaluated the utility of ex vivo cold IRI model with pan-caspase inhibitor (Z-VAD-FMK), which is effective in cold IRI using mice.

Methods: We subjected C57BL/6 mice to intracardiac injection of pan-caspase inhibitor or vehicle. After 24 hours of incubation in $4^{\circ} \mathrm{C}$ histidine-tryptophan-ketoglutarate solution, the kidney tissue was suspended on a 96 -well plate. So, incubation was performed at $37^{\circ} \mathrm{C}$ for 3,6 , and 9 hours, respectively. Then the kidneys were harvested for histology, western blot, reverse transcription polymerase chain reaction, and immunostaining to assess the effects of pan-caspase inhibitor.

Results: The pan-caspase inhibitor-treated group did not significantly improve histological tubular injury than that of the control group. However, pan-caspase inhibitor ameliorated the magnitude of an increase in kidney injury molecule- 1 levels in culture media at 3, 6, and 9 hours after rewarming and was significant at 6 hours. In addition, pan-caspase inhibitor did not show a decrease in proinflammatory cytokines but attenuated apoptosis in renal tubular cells.

Conclusions: Our data demonstrated that pan-caspase inhibitor reduced tubular injury in the ex vivo rewarming model after mouse kidney cold preservation.

Corresponding author: Sang-Ho Lee

E-mail: Ishkidney@khu.ac.kr

(c) The Korean Society for Transplantation

This is an Open Access article distributed under the terms of the Creative Commons Attribution Non-Commercial License (http://creativecommons.org/licenses/by-nc/4.0/) which permits unrestricted non-commercial use, distribution, and reproduction in any medium, provided the original work is properly cited. 